\section{Fibrosis sistémica nefrogénica: la pandemia que no fue}

\author{
CRISTIAN VARELA U., JUAN CARLOS PRIETO-RAYO
}

\section{Causal relationship between the use of gadolinium based contrast media and nephrogenic systemic fibrosis}

Nephrogenic systemic fibrosis (NSF) is a severe iatrogenic disease that affect patients with impaired renal function exposed to gadolinium-based contrast agents. Clinically, symptoms develop within days or weeks after the exposure and mimic a scleromyxedema. The causal relationship between use of gadoliniumbased contrast agents and NSF led to develop clinical guidelines aiming to limit the use of this contrast medium in high risk patients. These guidelines decreased the incidence of NSF in the last years. Unfortunately there is no specific treatment for NSF yet. Thus, strict adherence to current guidelines is key to prevent new cases. Renal dysfunction is increasingly common in our population. Therefore, practicing physicians should be aware of this potential complication of the use of gadolinium based contrast media.

(Rev Med Chile 2014; 142: 1565-1574)

Key words: Contrast media; Gadolinium; Magnetic resonance imaging; Nephrogenic fibrosing dermopathy; Renal insufficiency.

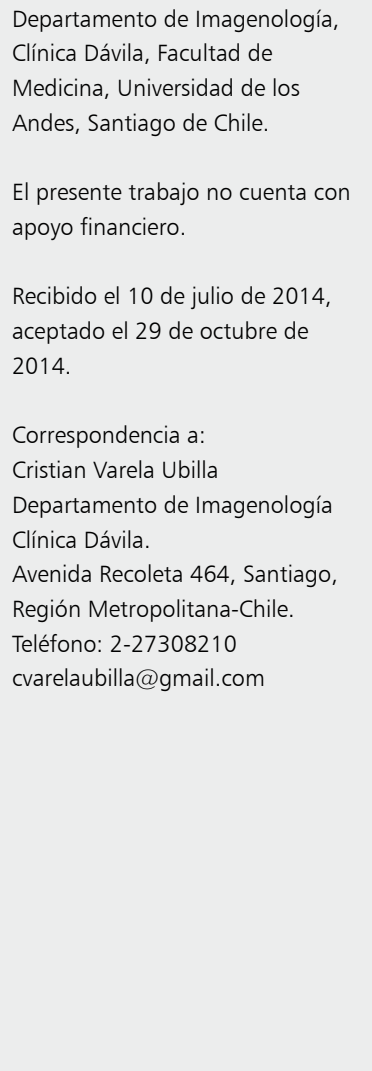

L a fibrosis sistémica nefrogénica (FSN) es una grave enfermedad iatrogénica que alarmó a la comunidad médica a partir de la descripción de la relación causal del uso de medios de contraste basados en gadolinio (MC-Gd) para resonancia magnética ( $\mathrm{RM})$, en pacientes con enfermedad renal avanzada, el año $2006^{1}$. Dado el uso masivo y universal de estos fármacos, hasta ese momento con un perfil de seguridad impecable, esta patología se perfilaba como una verdadera pandemia. Ocho años después y como un hecho casi inédito en la historia de la medicina, podemos afirmar que ha sido controlada con simples medidas de prevención ${ }^{2}$.

Se trata de una enfermedad poco frecuente, que hasta la fecha se ha desarrollado únicamente en pacientes con disminución severa de la función renal, ya sea aguda o crónica ${ }^{3}$. Clínicamente simula un esclero mixedema que se desarrolla en días o semanas ${ }^{4,5}$. Este artículo tiene como objetivo realizar una revisión actualizada de la FSN, revalorando su relevancia como complicación iatrogénica en pacientes con disfunción renal, con especial énfasis en los factores de riesgo y su prevención y por último conocer su realidad en Chile.

\section{Historia de la FSN}

Esta entidad clínica fue reconocida por primera vez en el año 1997 y descrita en el año 2000 por Cowper y cols. como una enfermedad cutánea escleromixedematosa-símil en pacientes con enfermedad renal crónica (ERC) en diálisis ${ }^{6}$. Inicialmente se pensaba que la enfermedad afectaba sólo la piel de los pacientes, por lo que se le llamó dermatopatía fibrosante nefrogénica. Posteriormente se comprobó la afección de múltiples órganos y sistemas por lo que en el año 2005 se propuso cambiar su nombre a $\mathrm{FSN}^{7}$.

En el año 2006, múltiples autores notaron una fuerte asociación entre la administración de MC- 
Gd utilizados en estudios diagnósticos con RM y la aparición de FSN en pacientes con enfermedad renal avanzada ${ }^{8,9}$.

Ese mismo año la U.S. Food and Drug Administration (FDA) hizo público un comunicado que advertía a pacientes y médicos sobre esta asociación ${ }^{10}$. A partir de este momento, organismos como el American College of Radiology (ACR), la FDA y la European Medicines Agency (EMA) tomaron medidas para regular la utilización de estos agentes en pacientes con daño renal, las cuales en el plazo de pocos años, generaron una marcada reducción de nuevos reportes de esta enfermedad (Figura 1) 2,11.

\section{Epidemiología}

La FSN es una condición poco frecuente. Se han reportado a la fecha alrededor de 500 casos en MedWatch (programa de reporte de efectos adversos de la FDA) y poco más de 380 casos en el registro de FSN de la Universidad de Yale ${ }^{12,13}$. Un artículo de revisión recolectó 408 casos confirmados con biopsia de FSN. Se reporta que la FSN se ha presentado en pacientes con edades desde 8 a 87 años, con un pico de incidencia entre los 51 y 60 años. No se demostró predilección racial o por género ${ }^{14}$.

\section{Factores de Riesgo}

El riesgo de FSN depende fundamentalmente de la función renal residual y del tipo y dosis de MC-Gd utilizado ${ }^{15-17}$.

\section{Función renal}

El deterioro de la función renal, tanto agudo como crónico, es considerado el mayor factor de riesgo para generar $\mathrm{FSN}^{18-20}$. No existe predilección por etiología o duración de la falla renal ${ }^{21,22}$.

Se estima que pacientes con ERC en etapa 5 (filtración glomerular $<15 \mathrm{ml} / \mathrm{min} / 1,73 \mathrm{~m}^{2}$ ) y etapa 4 (filtración glomerular entre $15 \mathrm{a} 29 \mathrm{ml} / \mathrm{min} / 1,73$ $\mathrm{m}^{2}$ ) tienen entre $1 \%$ y $7 \%$ de probabilidad de desarrollar FSN posterior a una o más exposiciones a algunos MC-Gd ${ }^{8,9,16,18,19,22,23}$. Sólo se ha publicado un reporte de caso en el cual el paciente desarrolló FSN con valores de filtración glomerular sobre 30 $\mathrm{ml} / \mathrm{min} / 1,73 \mathrm{~m}^{2} 24$. Entre $12 \%$ y $21 \%$ de los casos de FSN confirmados se desarrollaron en pacientes con injuria renal aguda (IRA), en la mayoría de estos casos existía a una ERC subyacente $e^{20,25,26}$.

\section{Gadolinio}

Es un metal utilizado como materia prima en diversas industrias. Por sus propiedades paramagnéticas se utiliza como medio de contraste en RM, ya que permite modificar el tiempo de relajación

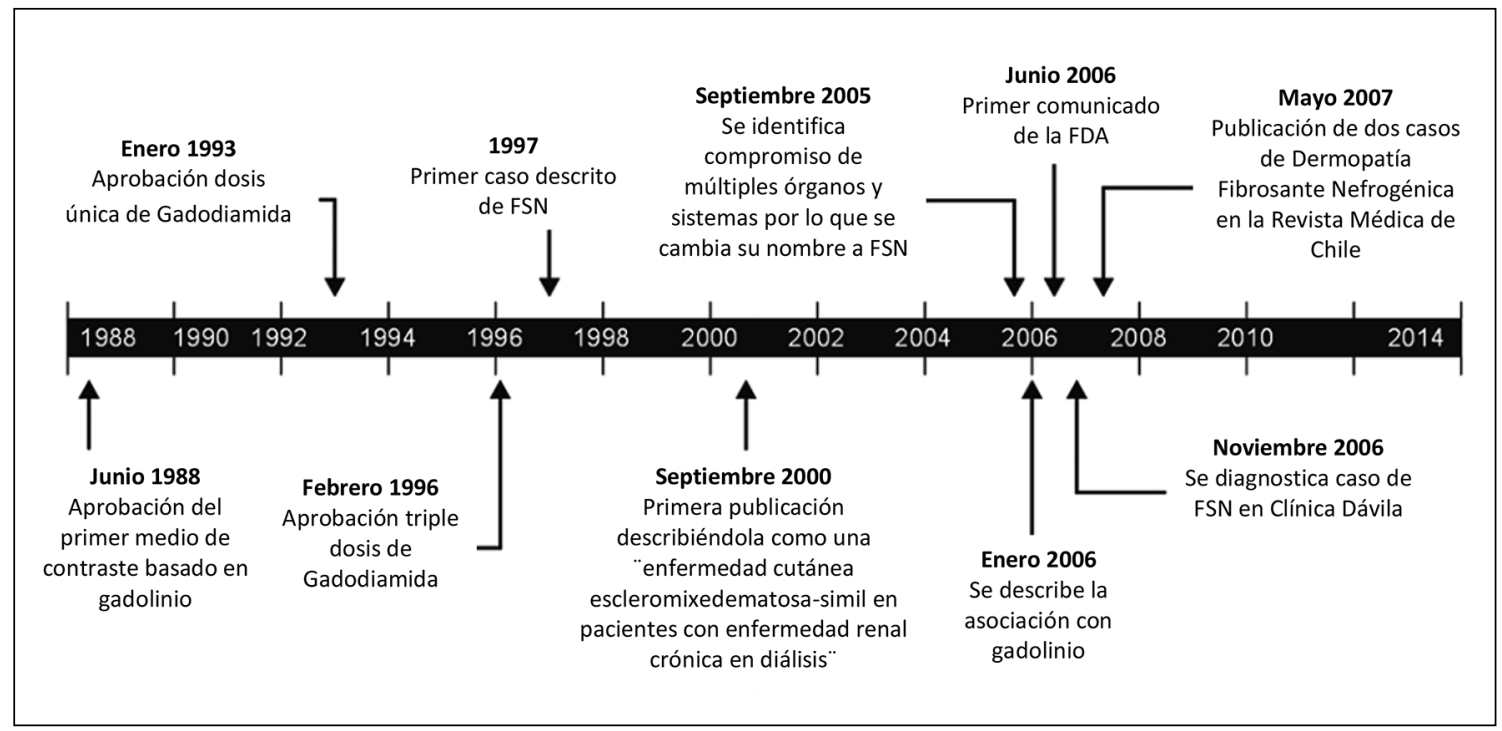

Figura 1. Línea de tiempo mostrando los principales hitos en la historia de la FSN. Modificado de referencia 1. 
de los tejidos y por ende mejorar el contraste de las imágenes adquiridas. En forma libre, el gadolinio es tóxico para el organismo, por lo que se administra unido a agentes quelantes. El primero de estos agentes fue aprobado por la FDA en el año 1988 para uso endovenoso ${ }^{1}$. Los principales usos de los MC-Gd son resaltar el contraste entre tejidos normales y patológicos, mejorando así la detección y caracterización de lesiones y en la angiografía por RM.

Hoy en día, la exposición a MC-Gd se considera un factor necesario para desarrollar FSN. El tiempo descrito entre la administración del MC-Gd y la aparición de los síntomas de FSN varía desde días a meses ${ }^{8,9,16,18,19,22}$. En casos excepcionales los síntomas aparecieron años después de la exposición al gadolinio ${ }^{18}$.

Al estudiar los MC-Gd involucrados en el desarrollo de la FSN, gadodiamida, gadoversetamida y gadopentetato, se observó que tenían en común la estructura molecular. Esta se caracteriza por una forma lineal (Figura 2a) la que permitiría una fácil liberación del gadolinio. Existen $\mathrm{MC}-\mathrm{Gd}$ que no fueron relacionados a la FSN como agentes etiológicos únicos. Estos poseen una molécula distinta, de tipo macrocíclica (Figura 2b) la cual contiene o "encierra" en su región central al gadolinio haciendo muy improbable su liberación. Se les considera agentes seguros en las distintas guías clínicas.

La gadodiamida fue el fármaco más utilizado en los casos reportados de FSN. Thomsen HS y cols. realizaron un estudio donde demostraron que la prevalencia de la NSF es significativamente mayor después de la administración de gadodiamida que cualquier otro MC-Gd (3-7\% vs $0-1 \%$ por inyección) en pacientes con función renal reducida ${ }^{29}$.

Estas diferencias respecto del perfil de seguridad entre los distintos MC-Gd llevó a que los comités de expertos de la ACR, EMA y la FDA a crear una clasificación según el riesgo de generar FSN (alto o grupo 1, intermedio o grupo 2 y bajo o grupo 3) con el objetivo de facilitar el uso de los mismos de acuerdo a las características de los pacientes $^{30}$. En la Tabla 1 se puede apreciar los diferentes MC-Gd y sus características.

Múltiples reportes sugieren una relación entre el riesgo de generar FSN y dosis de contraste ${ }^{3,22,26}$. Un estudio retrospectivo realizado por Broome y cols. analizó 301 pacientes que fueron sometidos a exámenes de RM con gadodiamida. De 207 pacientes que recibieron el doble de la dosis estándar $(0,2 \mathrm{mmol} / \mathrm{kg})$ de contraste, 12 pacientes $(5,7 \%)$ desarrollaron FSN, mientras que en los 94 pacientes que recibieron una dosis estándar de MC $(0,1 \mathrm{mmol} / \mathrm{kg})$, no se observó desarrollo de esta patología ${ }^{22}$. Sin embargo, algunos casos de FSN se desarrollaron después de la administración de dosis única y estándar de contraste $e^{17,18,23}$.

\section{Otros factores}

Es evidente que no todos los pacientes con deterioro de la función renal expuestos a MC-Gd desarrollan FSN. Se han observado una serie de condiciones que podrían actuar como factores permisivos en el desarrollo de esta patología. Estos incluyen condiciones proinflamatorias y alteraciones bioquímicas. Dentro de los primeros se identifican: cirugía vascular reciente, eventos protrombóticos o estados procoagulantes, infecciones severas e hiperparatiroidismo $\mathrm{s}^{5,32,33}$. Entre las

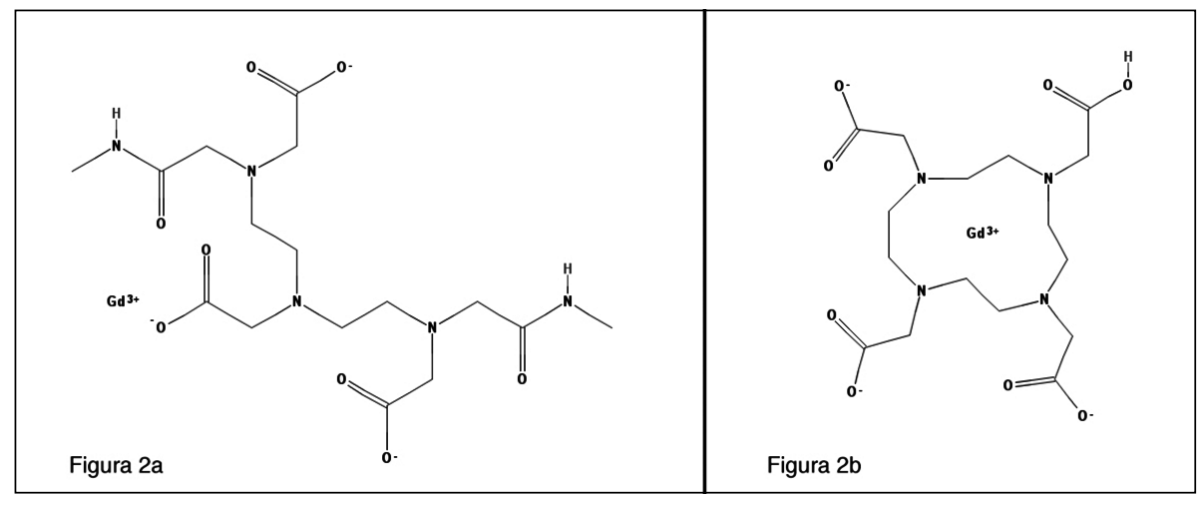

Figura 2. Estructura molecular de distintos MC-Gd. La Figura 2a corresponde a la molécula lineal de gadodiamida, mientras que la Figura $\mathbf{2 b}$ corresponde a una molécula macrocíclica de gadoterato. Observe como el gadolinio queda "encerrado" por la molécula quelante en el segundo caso $^{27,28}$. 
Tabla 1. Medios de contraste basados en gadolinio y sus características

\begin{tabular}{|c|c|c|c|c|c|c|}
\hline & Nombre & $\begin{array}{l}\text { Nombre } \\
\text { comercial }\end{array}$ & Quelante/carga & Estabilidad & $\begin{array}{l}\text { Incidencia } \\
\text { de FSN* }\end{array}$ & $\begin{array}{c}\text { Aprobación } \\
\text { FDA }\end{array}$ \\
\hline \multirow[t]{3}{*}{1} & Gadodiamida** & Omniscan $®$ & Lineal/no iónico & Baja & Alta & 1993 \\
\hline & Gadoversetamida** & OptiMARK® & Lineal/no iónico & Baja & Alta & 1999 \\
\hline & Gadopentetato de dimeglumina*** & Magnevistan $®$ & Lineal/iónico & Intermedia & Alta & 1988 \\
\hline \multirow[t]{3}{*}{2} & Gadobenato de dimeglumina & MultiHance ${ }^{\circledR}$ & Lineal/iónico & Intermedia & Intermedia & 2004 \\
\hline & Gadoxetato disódico & $\begin{array}{l}\text { Primovist } \AA \\
\text { Eovist } \AA\end{array}$ & Lineal/iónico & Intermedia & Intermedia & 2008 \\
\hline & Gadofosveset trisódico & $\begin{array}{l}\text { Vasovist }{ }^{\circledR} \\
\text { Ablavar }\end{array}$ & Lineal/iónico & Intermedia & Intermedia & 2008 \\
\hline \multirow[t]{3}{*}{3} & Gadobutrol ${ }^{* * *}$ & $\begin{array}{l}\text { Gadovist }{ }^{\circledR} \\
\text { Gadavist }{ }^{\circledR}\end{array}$ & $\begin{array}{l}\text { Macrocíclico/ } \\
\text { no iónico }\end{array}$ & Alta & Baja & 2011 \\
\hline & Gadoteridol & ProHance ${ }^{\circledR}$ & $\begin{array}{l}\text { Macrocíclico/ } \\
\text { no iónico }\end{array}$ & Alta & Baja & 1992 \\
\hline & Gadoterato de Meglumina** & $\begin{array}{l}\text { Dotarem }{ }^{\circledR} \\
\text { Magnescope } \AA\end{array}$ & $\begin{array}{l}\text { Macrocíclico/ } \\
\text { iónico }\end{array}$ & Alta & Baja & 2013 \\
\hline
\end{tabular}

Modificado de referencia 3. *Según clasificación EMA ${ }^{30}$. **Fármacos disponibles en Chile ${ }^{31}$. ***Fármaco aprobado recientemente en Chile ${ }^{31}$. ${ }^{* * *}$ Fármaco utilizado en Chile, actualmente no vigente en listado del ISP31. FSN: Fibrosis Sistémica Nefrogénica. FDA: US Food and Drug Administration. EMA: European Medicines Agency.

alteraciones bioquímicas destacan: acidosis metabólica, niveles elevados de hierro, calcio y/o fosfato y terapia con dosis altas de eritropoyetina ${ }^{8,25,34,35}$.

\section{Fisiopatología}

El mecanismo exacto mediante el cual se desarrolla la FSN no se comprende del todo. Pacientes sanos eliminan rápidamente el complejo gadolinio-quelante indemne fundamentalmente vía renal. En pacientes con función renal disminuida, en cambio, el gadolinio permanece largo tiempo en el organismo pudiendo disociarse del agente quelante. Por ejemplo, gadodiamida tiene una vida media de $2 \mathrm{~h}$ en individuos sanos con función renal normal y aumenta a alrededor de $30 \mathrm{~h}$ en pacientes con ERC etapa $5^{36}$. El gadolinio libre puede unirse a aniones disponibles en el medio como fosfato, formando sales insolubles y depositándose en los tejidos ${ }^{37}$. Se ha detectado depósitos de gadolinio en biopsias de piel $^{38,39}$ y otros tejidos de pacientes con $\mathrm{FSN}^{40,41}$, pero no en la piel de individuos sanos expuestos a MC basados en gadolinio ${ }^{42}$.

Se desconoce el mecanismo exacto mediante el cual el gadolinio se deposita en los tejidos, pero existe evidencia que orienta a que eventos inflamatorios o trombóticos pueden alterar la permeabilidad vascular endotelial ${ }^{8,16,33,43}$.
Se han realizado varios estudios, la mayoría in vitro, tratando de encontrar el mecanismo mediante el cual se induce fibrosis. Gadodiamida podría unirse a receptores Toll-like (TLR) en monocitos o macrófagos y estimular la producción de citoquinas profibróticas y proinflamatorias ${ }^{44}$. Las moléculas producidas incluyen interleuquina (IL) -4, IL-6, IL-13, interferón (IFN) $-\gamma$, factor de crecimiento endotelial vascular (VEGF) y factor de crecimiento transformante beta (TGF- $\beta$ ), además de citoquinas dependientes del factor nuclear potenciador de las cadenas ligeras kappa de las células $B$ activadas (NF- $\kappa B$ ) como CCL2, CCL8, CXCL10, CXCL11 y CXCL12 ${ }^{44,45}$. Al incubar monocitos con cloruro de gadolinio se producen citoquinas $y$ factores de crecimiento similares, lo que orienta a que la estimulación se debe al gadolinio libre en lugar del complejo gadolinio-quelante ${ }^{43}$. Por otra parte, el gadolinio puede inducir apoptosis en macrófagos ${ }^{46}$.

Se cree que la liberación de estos mediadores atraería fibrocitos circulantes que expresan CD34 y procolágeno- $1^{47}$. Una vez en el tejido alterado, los fibrocitos se diferencian a miofibroblastos, los cuales contribuirían al desarrollo de la FSN. Por otra parte, el medio de cultivo donde se incubaron monocitos de sangre periférica con gadodiamida, gadopentetato o cloruro de gadolinio, estimuló la producción y secreción de procolágeno-1 y la 
expresión de $\alpha$-actina de músculo liso ( $\alpha$-SMA) en fibroblastos de la piel ${ }^{43}$.

Se ha comprobado que tanto gadodiamida como gadopentetato pueden estimular la producción de colágeno tipo 1 por los fibroblastos ${ }^{48}$. Otro estudio in vitro demostró que gadodiamida disminuía el tiempo de duplicación de los fibroblastos de $28 \mathrm{~h}$ a $22 \mathrm{~h}$, lo que sugiere que dicho compuesto genera activación celular ${ }^{49}$. Resultados similares se observaron con cloruro de gadolinio en bajas concentraciones ${ }^{50}$.

El mecanismo por el cual se produce la estimulación de los fibroblastos es complejo. Bhagavathula y cols. realizaron un estudio sugiriendo que tanto la vía de las MAP-kinasas como la vía del fosfoinositol 3-quinasa (PI3K) estarían implicadas en la activación de fibroblastos por gadolinio ${ }^{51}$.

\section{Manifestaciones clínicas}

La FSN se presenta típicamente como un engrosamiento, endurecimiento e hiperpigmentación progresiva de la piel, principalmente de las extremidades. Las alteraciones cutáneas generalmente se inician de forma simétrica en las extremidades inferiores y progresan hacia cefálico. La piel del tronco se ve afectada con mucha menos frecuencia que la de las extremidades y se respeta el territorio facial ${ }^{52}$.

En la fase temprana del curso de la FSN, la piel afectada puede estar eritematosa y edematosa, sin hiperpigmentación. Estas lesiones son muy pruriginosas y pueden ser confundidas con reacciones alérgicas. A medida que la fibrosis progresa la piel gradualmente se adhiere a la fascia subyacente al punto de no poder ser traccionada. Estas lesiones suelen ser extremadamente dolorosas ${ }^{53}$.

Otra manifestación cutánea de esta enfermedad es la aparición de placas, rojas o violáceas, con proyecciones ameboideas digitiformes o reticulares (Figura 3). En estados más avanzados los pacientes pueden generar atrofia epidérmica, "empedrado", pérdida de vello o piel de naranja. Estos cambios conducen a disminución del rango articular de codos, rodillas y dedos, con discapacidad progresiva que puede llegar a ser severa (Figura 4$)^{54,55}$.

La aparición de nódulos o placas amarillas en las escleras es otro signo sugerente. Al igual que el compromiso cutáneo puede motivar al paciente a consultar. La presencia de lesiones de esclera constituye un criterio menor para el diagnóstico de FSN, especialmente en menores de 45 años $^{55}$.

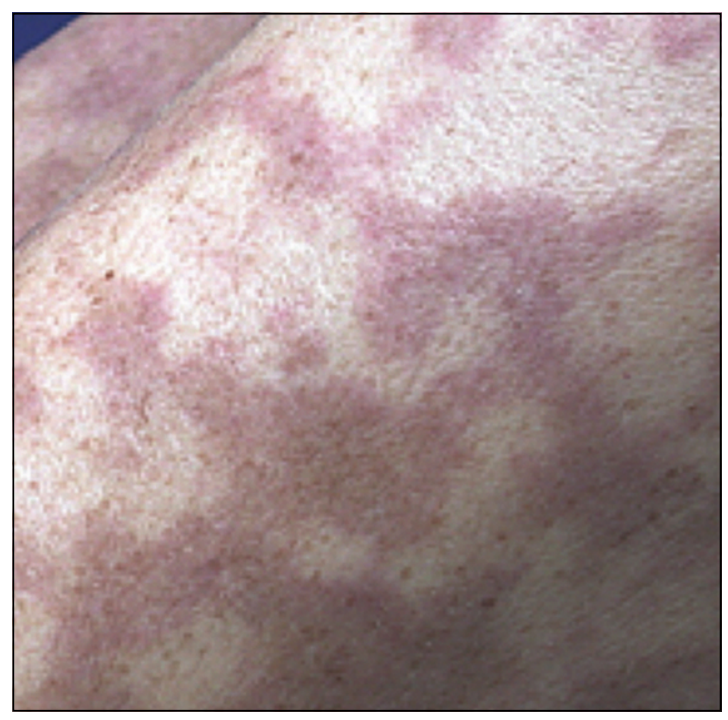

Figura 3. Placas eritematosas con proyecciones ameboideas correspondientes a estados iniciales de la enfermedad. Reproducido de: Girardi M, et al. $2011^{55}$. Copyright 2014. Usado con permiso de Elsevier/American Academy of Dermatology, Inc.

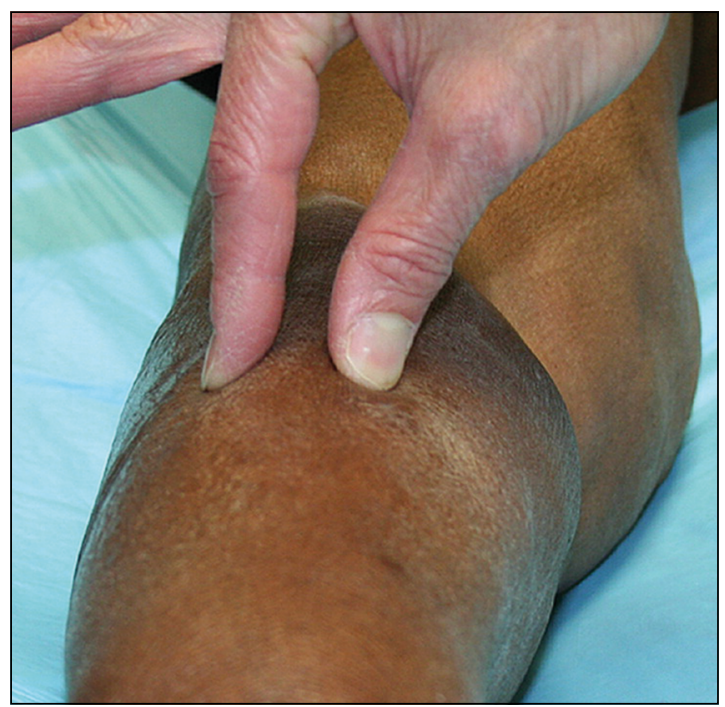

Figura 4. La imagen muestra el compromiso cutáneo en estados avanzados de la enfermedad. Observe el endurecimiento y adherencia de la piel a la fascia subyacente, la cual no permite ser traccionada. Reproducido de: Girardi M, et al. 201155. Copyright 2014. Usado con permiso de Elsevier/ American Academy of Dermatology, Inc. 
Además del compromiso cutáneo y escleral se ha confirmado el desarrollo de fibrosis en músculos esqueléticos, diafragma, linfonodos, corazón, pulmones, esófago, hígado, tracto genitourinario y duramadre ${ }^{40,41,56}$.

\section{Pronóstico}

Esta patología es progresiva en la mayoría de los casos. Algunos reportes sugieren que el restablecimiento de la función renal parece detener o enlentecer la progresión de la FSN. Este beneficio se observó en pacientes ERC que fueron sometidos a trasplante renal de forma exitosa y pacientes con IRA cuya función renal se recuperó ${ }^{57}$.

La patología cardiovascular es la principal causa de muerte en los pacientes con enfermedad renal, ya sea con o sin FSN. La FSN no es letal de forma directa, pero se ha visto que los pacientes con ERC en etapa 5 con cambios cutáneos de NSF tienen un riesgo tres veces mayor de morir dentro de los 2 años del diagnóstico de la NSF versus los que no presentan $\mathrm{FSN}^{17,58}$.

A pesar de que esta enfermedad no representa un riesgo vital inmediato, constituye una condición limitante con un gran impacto en la calidad de vida. La reducción de la movilidad determina mayor riesgo de caídas y fracturas lo que se traduce en mayor morbimortalidad ${ }^{52}$.

\section{Tratamiento}

No existe tratamiento específico para esta patología. Múltiples terapias han sido propuestas pero ninguna ha mostrado ser tan efectiva como la restauración de la función renal. Entre ellas destacan terapias destinadas a eliminar o bloquear el MC-Gd circulante e inmunomoduladores (fotoferesis extracorpórea ${ }^{59}$, plasmaferesis ${ }^{60}$, tiosulfato de sodio ${ }^{61}$ y mesilato de imatinib ${ }^{62}$ ). La fisioterapia ha mostrado utilidad en casos de rigidez articular, permitiendo mantener la movilidad ${ }^{63}$.

\section{Prevención}

La identificación de los pacientes en riesgo ha logrado reducir de forma sostenida los nuevos casos de $\mathrm{FSN}^{11}$. El comité de expertos de la ACR recomienda considerar como pacientes en riesgo de desarrollar FSN los que cumplan con cualquiera de las siguientes condiciones: 1) Paciente en diálisis, en cualquiera de sus formas; 2) ERC en etapa 4 ó 5 sin diálisis; 3 ) ERC en etapa 3 sin diálisis (esto porque su función renal puede fluctuar) y 4) pacientes con IRA ${ }^{64}$.

Para este grupos de pacientes deben considerarse procedimientos diagnósticos alternativos que no involucren MC basados en gadolinio (ecotomografía o tomografía computada). Cuando el beneficio de realizar el examen supere al potencial riesgo o no exista un test alternativo equivalente, se debe informar tanto al médico que solicitó el examen como al paciente de las posibles consecuencias y ambos deben estar de acuerdo. Se recomienda firmar un consentimiento informado. También se recomienda administrar un MC-Gd macrocíclico del grupo 3, los agentes del grupo 1 están contraindicados en forma absoluta. Además se debe administrar la menor dosis posible de MC-Gd que permita generar las imágenes necesarias para el diagnóstico. Es necesario registrar el nombre y dosis del fármaco utilizado. También se recomienda evitar la administración de múltiples dosis de MC-Gd en períodos de tiempo reducidos. La European Society for Urological Radiology (ESUR) recomienda esperar un tiempo no menor a una semana entre inyecciones ${ }^{30}$.

En pacientes anúricos sin función renal residual que requieran un estudio contrastado se debe considerar la administración de contraste yodado y realizar una tomografía computada en vez de una RM con MC-Gd.

Otra recomendación propuesta por la ACR consiste en realizar el examen contrastado con MC-Gd inmediatamente antes de la sesión de hemodiálisis del paciente, esta recomendación se basa sólo en la opinión de expertos, sin trabajos científicos que prueben su efectividad.

\section{Realidad en Chile}

En nuestro país la RM es una técnica madura que se practica rutinariamente desde hace al menos tres décadas y está disponible tanto en el sistema privado como en varios hospitales públicos. No existen cifras oficiales disponibles, sin embargo, es posible afirmar que se realizan varios cientos de estudios al día y probablemente entre 30 y $50 \%$ de ellos se utilizan MC-Gd.

Según el registro del Instituto de Salud Pública (ISP) existen cinco MC-Gd inscritos y aprobados para su uso en nuestro país ${ }^{31}$. Omiscan $^{\circledR}$, OptiMARK ${ }^{\circledR}$ y Dotarem ${ }^{\circledR}$ están vigentes 
y son usados ampliamente en nuestra realidad. Magnevistan ${ }^{\circledR}$ no ha renovado su situación en el ISP, por lo que no se comercializa actualmente en Chile. Gadovist ${ }^{\circledR}$ ha sido inscrito recientemente pero todavía no está disponible como producto comercial.

De estos fármacos Omiscan ${ }^{\circledR}$ (gadodiamida) y OptiMARK ${ }^{\circledR}$ (gadoversetamida), corresponden a agentes del grupo 1. Según normas internacionales ambos fármacos están contraindicados en pacientes en riesgo. Como fue mencionado, ante la eventualidad de tener que utilizar MC-Gd en estos pacientes, se deben administrar fármacos del grupo 3 como Dotarem ${ }^{\circledR}$ (gadoterato de meglumina) y Gadovist ${ }^{\circledR}$ (gadobutrol).

Desafortunadamente existen casos de FSN en nuestro país. Uno de estos fue diagnosticado en la unidad de diálisis del Departamento de Nefrología de Clínica Dávila el año 2006. Corresponde a un hombre de 32 años con ERC de un año y medio de evolución de causa desconocida. En febrero del año 2006 se le practicó una RM abdominal con gadodiamida. En noviembre del mismo año se realizó una angio-RM para descartar TEP utilizando nuevamente gadodiamida. Veinte días después consultó por severo dolor en extremidades inferiores asociado a mialgias, artralgias y rigidez articular. Al examen físico destacó la presencia de lesiones caracterizadas por endurecimiento cutáneo en región pretibial, con oscurecimiento de la piel y edema que no deja fóvea. Se descartaron enfermedades autoinmunes y se inició terapia corticoesteroidal empírica por dos meses sin resultados. Se realizó biopsia de lesiones de piel en región pretibial, cuyo resultado fue compatible con FSN.

El paciente evolucionó con mal control del dolor, rigidez articular e invalidez progresiva que lo lleva a postración. Fue asistido por equipo multidisciplinario con fisiatría, psiquiatría y terapia de dolor. Posteriormente sufrió caída y fractura de cadera que requería cirugía. Falleció el año 2008 como consecuencia de falla cardiaca.

En mayo de 2007 el Departamento de Dermatología de la Pontificia Universidad Católica de Chile publicó en la Revista Médica de Chile dos casos de dermatopatía fibrosante nefrogénica ${ }^{65}$. En dicha publicación existe mención de la recientemente descrita relación causal con el uso de MC-Gd, sin embargo, no explicita si sus pacientes recibieron o no estos productos.

\section{Conclusión}

La FSN es una grave complicación de la administración de MC-Gd, en pacientes con disminución de la función renal. La descripción de dicha relación causal derribó el impecable perfil de bioseguridad de estos agentes, que hasta ese entonces eran recomendados en pacientes con insuficiencia renal por su escasa o nula nefrotoxicidad.

Las advertencias y recomendaciones iniciales emitidas por organismos reguladores como la FDA, fueron seguidas de guías clínicas que han tenido amplia difusión en el uso restrictivo de estos agentes. Como consecuencia en pocos años se logró una marcada reducción en la incidencia de esta entidad.

Como médicos al cuidado de pacientes cada vez más añosos y complejos, en los que el deterioro de la función renal es frecuente, debemos estar en conocimiento de esta rara pero potencialmente grave complicación del uso de MC-Gd. El apego estricto a guías clínicas de prevención será fundamental para evitar nuevos casos.

Agradecimientos: Agradecemos la generosidad de la Dra. Marcela Ursu, nefróloga del Departamento de Nefrología de Clínica Dávila al proveer información sobre el caso descrito en el artículo.

\section{Referencias}

1. Juluru K, Vogel-Claussen J, Macura KJ, Kamel IR, Steever $A$, Bluemke DA. MR imaging in patients at risk for developing nephrogenic systemic fibrosis: Protocols, practices, and imaging techniques to maximize patient safety. Radiographics 2009; 29 (1): 9-22.

2. Wang Y, Alkasab TK, Narin O, Nazarian RM, Kaewlai R, Kay J, et al. Incidence of nephrogenic systemic fibrosis after adoption of restrictive gadolinium-based contrast agent guidelines. Radiology 2011; 260 (1): 105-11.

3. Daftari Besheli L, Aran S, Shaqdan K, Kay J, Abujudeh H. Current status of nephrogenic systemic fibrosis. Clin Radiol 2014; 69 (7): 661-8.

4. Scheinfeld N. Nephrogenic fibrosing dermopathy: a comprehensive review for the dermatologist. Am J Clin Dermatol 2006; 7 (4): 237-47.

5. Cowper SE, Rabach M, Girardi M. Clinical and histological findings in nephrogenic systemic fibrosis. Eur J Radiol 2008; 66 (2): 191-9.

6. Cowper SE, Robin HS, Steinberg SM, Su LD, Gupta S, LeBoit PE. Scleromyxoedema-like cutaneous diseases in 
renal-dialysis patients. Lancet 2000; 356 (9234): 1000-1.

7. Gibson SE, Farver CF, Prayson RA. Multiorgan involvement in nephrogenic fibrosing dermopathy: an autopsy case and review of the literature. Arch Pathol Lab Med 2006; 130 (2): 209-12.

8. Grobner T. Gadolinium-a specific trigger for the development of nephrogenic fibrosing dermopathy and nephrogenic systemic fibrosis? Nephrol Dial Transplant 2006; 21 (4): 1104-8.

9. Marckmann P, Skov L, Rossen K, Dupont A, Damholt MB, Heaf JG, et al. Nephrogenic systemic fibrosis: suspected causative role of gadodiamide used for contrast-enhanced magnetic resonance imaging. J Am Soc Nephrol 2006; 17 (9): 2359-62.

10. U.S. Food and Drug Administration A Public Health Advisory. Gadolinium-containing contrast agents for magnetic resonance imaging (MRI). 2006. Disponible en: http://www.fda.gov/Drugs/DrugSafety/PostmarketDrugSafetyInformationforPatientsandProviders/ DrugSafetyInformationforHeathcareProfessionals/ PublicHealthAdvisories/ucm053112.htm (accedido en junio de 2014).

11. Altun E, Martin DR, Wertman R, Lugo-Somolinos A, Fuller ER 3rd, Semelka RC. Nephrogenic systemic fibrosis: change in incidence following a switch in gadolinium agents and adoption of a gadolinium policyreport from two U.S. universities. Radiology 2009; 253 (3): 689-96.

12. Braverman I, Cowper SE Nephrogenic systemic fibrosis. F1000 Med Rep 2010; 2: 84.

13. Cowper SE. Nephrogenic Systemic Fibrosis [ICNSFR Website]. 2001-2013. Disponible en http://www.icnsfr. org. Visitado en junio de 2014.

14. Zou Z, Ma L Nephrogenic systemic fibrosis: review of 408 biopsy-confirmed cases. Indian J Dermatol 2011; 56 (1): 65-73

15. Rydahl C, Thomsen HS, Marckmann P. High prevalence of nephrogenic systemic fibrosis in chronic renal failure patients exposed to gadodiamide, agadolinium-containing magnetic resonance contrast agent. Invest Radiol 2008; 43 (2): 141-4.

16. Sadowski EA, Bennett LK, Chan MR, Wentland AL, Garrett AL, Garrett RW, et al. Nephrogenic systemic fibrosis: risk factors and incidence estimation. Radiology 2007; 243 (1): 148-57.

17. Todd DJ, Kagan A, Chibnik LB, Kay J. Cutaneous changes of nephrogenic systemic fibrosis: predictor of early mortality and association with gadolinium exposure. Arthritis Rheum 2007; 56 (10): 3433-41.

18. Shabana WM, Cohan RH, Ellis JH, Hussain HK, Francis IR, Su LD, et al. Nephrogenic systemic fibrosis: a report of 29 cases. AJR Am J Roentgenol 2008; 190 (3): 736-41.

19. Wertman R, Altun E, Martin DR, Mitchell DG, Leyendecker JR, O’Malley RB, et al. Risk of nephrogenic systemic fibrosis: evaluation of gadolinium chelate contrast agents at four American universities. Radiology 2008; 248 (3): 799-806.

20. Pérez-Rodríguez J, Lai S, Ehst BD, Fine DM, Bluemke DA. Nephrogenic systemic fibrosis: incidence, associations, and effect of risk factor assessment-report of 33 cases. Radiology 2009; 250 (2): 371-7.

21. Abujudeh HH, Kaewlai R, Kagan A, Chibnik LB, Nazarian RM, High WA, et al. Nephrogenic systemic fibrosis after gadopentetate dimeglumine exposure: case series of 36 patients. Radiology 2009; 253 (1): 81-9.

22. Broome DR, Girguis MS, Baron PW, Cottrell AC, Kjellin I, Kirk GA. Gadodiamide-associated nephrogenic systemic fibrosis: why radiologists should be concerned. AJR Am J Roentgenol 2007; 188 (2): 586-92.

23. Collidge TA, Thomson PC, Mark PB, Traynor JP, Jardine AG, Morris ST, et al. Gadolinium-enhanced MR imaging and nephrogenic systemic fibrosis: retrospective study of a renal replacement therapy cohort. Radiology 2007; 245 (1): 168-75.

24. Shibui K, Kataoka H, Sato N, Watanabe Y, Kohara M, Mochizuki T. A case of NSF attributable to contrast MRI repeated in a patient with Stage $3 \mathrm{CKD}$ at a renal function of eGFR $>30 \mathrm{ml} / \mathrm{min} / 1,73 \mathrm{~m}^{2}$. Jpn J Nephrol 2009; 51: 676.

25. Abu-Alfa AK. Nephrogenic systemic fibrosis and gadolinium-based contrast agents. Adv Chronic Kidney Dis 2011; 18 (3): 188-98.

26. Prince $\mathrm{MR}$, Zhang $\mathrm{H}$, Morris $\mathrm{M}$, MacGregor JL, Grossman ME, Silberzweig J, et al. Incidence of nephrogenic systemic fibrosis at two large medical centers. Radiology 2008; 248 (3): 807-16.

27. National Center for Biotechnology Information. PubChem Compound Database; CID=153921, https:// pubchem.ncbi.nlm.nih.gov/summary/summary. cgi?cid=153921 (accedido en junio de 2014).

28. National Center for Biotechnology Information. PubChem Compound Database; CID $=6918037$. https:// pubchem.ncbi.nlm.nih.gov/summary/summary. cgi?cid=6918037 (accedido en junio de 2014).

29. Thomsen HS, Marckmann P. Extracellular Gd-CA: differences in prevalence of NSF. Eur J Radiol 2008; 66 (2): 180-3.

30. Thomsen HS, Morcos SK, Almén T, Bellin MF, Bertolotto M, Bongartz G, et al. Nephrogenic systemic fibrosis and gadolinium-based contrast media: updated ESUR Contrast Medium Safety Committee guidelines. Eur Radiol 2013; 23 (2): 307-18. 
31. Agencia Nacional de Medicamentos (ANAMED), Instituto de Salud Pública de Chile. http://registrosanitario. ispch.gob.cl/ (accedido en junio de 2014).

32. Cowper SE. Nephrogenic systemic fibrosis: an overview. J Am Coll Radiol 2008; 5 (1): 23-8.

33. Chopra T, Kandukurti K, Shah S, Ahmed R, Panesar M. Understanding nephrogenic systemic fibrosis. Int J Nephrol 2012; 2012: 912189.

34. Peak AS, Sheller A. Risk factors for developing gadolinium-induced nephrogenic systemic fibrosis. Ann Pharmacother 2007; 41 (9): 1481-5.

35. High WA, Ayers RA, Chandler J, Zito G, Cowper SE. Gadolinium is detectable within the tissue of patients with nephrogenic systemic fibrosis. J Am Acad Dermatol 2007; 56: 21-6.

36. Joffe P, Thomsen HS, Meusel M. Pharmacokinetics of gadodiamide injection in patients with severe renal insufficiency and patients undergoing hemodialysis or continuous ambulatory peritoneal dialysis. Acad Radiol 1998; 5 (7): 491-502.

37. Baranyai Z, Pálinkás Z, Uggeri F, Maiocchi A, Aime S, Brücher E. Dissociation kinetics of open-chain and macrocyclic gadolinium (iii)-aminopolycarboxylate complexes related to magnetic resonance imaging: catalytic effect of endogenous ligands. Chemistry 2012; 18 (51): 16426-35.

38. High WA, Ayers RA, Chandler J, Zito G, Cowper SE. Gadolinium is detectable within the tissue of patients with nephrogenic systemic fibrosis. J Am Acad Dermatol 2007; 56 (1): 21-6.

39. Boyd AS, Zic JA, Abraham JL. Gadolinium deposition in nephrogenic fibrosing dermopathy. J Am Acad Dermatol 2007; 56 (1): 27-30.

40. Kay J, Bazari H, Avery LL, Koreishi AF. Case records of the Massachusetts General Hospital. Case 6-2008. A 46-year-old woman with renal failure and stiffness of the joints and skin. N Engl J Med 2008; 358 (8): 827-38.

41. Sanyal S, Marckmann P, Scherer S, Abraham JL. Multiorgan gadolinium (Gd) deposition and fibrosis in a patient with nephrogenic systemic fibrosis-an autopsy-based review. Nephrol Dial Transplant 2011; 26 (11): 3616-26.

42. Boyd AS, Sanyal S, Abraham JL. Gadolinium is not deposited in the skin of patients with normal renal function after exposure to gadolinium-based contrast agents. J Am Acad Dermatol 2008; 59 (2): 356-8.

43. Wermuth PJ, Del Galdo F, Jiménez SA. Induction of the expression of profibrotic cytokines and growth factors in normal human peripheral blood monocytes by gadolinium contrast agents. Arthritis Rheum 2009; 60 (5): 1508-18.

44. Wermuth PJ, Jiménez SA. Gadolinium compounds signaling through TLR 4 and TLR 7 in normal human macrophages: establishment of a proinflammatory phenotype and implications for the pathogenesis of nephrogenic systemic fibrosis. J Immunol 2012; 189 (1): 318-27.

45. Del Galdo F, Wermuth PJ, Addya S, Fortina P, Jiménez SA. NFkB activation and stimulation of chemokine production in normal human macrophages by the gadolinium-based magnetic resonance contrast agent Omniscan: possible role in the pathogenesis of nephrogenic systemic fibrosis. Ann Rheum Dis 2010; 69 (11): 2024-33.

46. Mizgerd JP, Molina RM, Stearns RC, Brain JD, Warner AE. Gadolinium induces macrophage apoptosis. J Leukoc Biol 1996; 59 (2): 189-95.

47. Bucala R. Circulating fibrocytes: cellular basis for NSF. J Am Coll Radiol 2008; 5 (1): 36-9.

48. Piera-Velazquez S, Louneva N, Fertala J, Wermuth PJ, Del Galdo F, Jiménez SA. Persistent activation of dermal fibroblasts from patients with gadolinium-associated nephrogenic systemic fibrosis. Ann Rheum Dis 2010; 69 (11): 2017-23.

49. Edward M, Quinn JA, Mukherjee S, Jensen MB, Jardine AG, Mark PB, et al. Gadodiamide contrast agent "activates" fibroblasts: a possible cause of nephrogenic systemic fibrosis. J Pathol 2008; 214 (5): 584-93.

50. Abraham JL, Edward M. Free gadolinium is a likely trigger of nephrogenic systemic fibrosis. AJR Am J Roentgenol 2009; 193 (4): 354.

51. Bhagavathula N, DaSilva M, Aslam MN, Dame MK, Warner RL, Xu Y, et al. Regulation of collagen turn over in human skin fibroblasts exposed to a gadoliniumbased contrast agent. Invest Radiol 2009; 44 (8): 433-9.

52. Igreja AC, Mesquita K de C, Cowper SE, Costa IM. Nephrogenic systemic fibrosis: concepts and perspectives. An Bras Dermatol 2012; 87 (4): 597-607.

53. Bernstein EJ, Schmidt-Lauber C, Kay J. Nephrogenic systemic fibrosis: a systemic fibrosing disease resulting from gadolinium exposure. Best Pract Res Clin Rheumatol 2012; 26 (4): 489-503.

54. Moschella SL, Kay J, Mackool BT, Liu V. Case records of the Massachusetts General Hospital. Weekly clinicopathological exercises. Case 35-2004. A 68-year-old man with end-stage renal disease and thickening of the skin. N Engl J Med 2004; 351 (21): 2219-27.

55. Girardi M, Kay J, Elston DM, LeBoit PE, Abu-Alfa A, Cowper SE. Nephrogenic Systemic Fibrosis: Clinicopathological definition and workup recommendations. J Am Acad Dermatol 2011; 65 (6): 1095-1106.e7.

56. Koreishi AF, Nazarian RM, Saenz AJ, Klepeis VE, McDonald AG, Farris AB, et al. Nephrogenic systemic fibrosis: 
a pathologic study of autopsy cases. Arch Pathol Lab Med 2009; 133 (12): 1943-8.

57. Cuffy MC, Singh M, Formica R, Simmons E, Abu Alfa AK, Carlson K, et al. Renal transplantation for nephrogenic systemic fibrosis: a case report and review of the literature. Nephrol Dial Transplant 2011; 26 (3): 1099101.

58. Basak P, Jesmajian S. Nephrogenic systemic fibrosis: current concepts. Indian J Dermatol 2011; 56 (1): 59-64.

59. Mathur K, Morris S, Deighan C, Green R, Douglas KW. Extracorporeal photopheresis improves nephrogenic fibrosing dermopathy/nephrogenic systemic fibrosis: three case reports and review of literature. J Clin Apheresis 2008; 23 (4): 144-50.

60. Baron PW, Cantos K, Hillebrand DJ, Hu KQ, Ojogho ON, Nehlsen-Cannarella S, et al. Nephrogenic fibrosing dermopathy after liver transplantation successfully treated with plasmapharesis. Amer J Dermopathol 2003; 25
(3): 204-9.

61. Kadiyala D, Roer DA, Perazella MA. Nephrogenic systemic fibrosis associated with gadoversetamide exposure: treatment with sodium thiosulfate. Am J Kidney Dis 2009; 53 (1): 133-7.

62. Kay J, High WA. Imatinib mesylate treatment of nephrogenic systemic fibrosis. Arthritis Rheum 2008; 58 (8): 2543-8.

63. Linfert DR, Schell JO, Fine DM. Treatment of nephrogenicsy stemic fibrosis: limited options but hope for the future. Semin Dial 2008; 21 (2): 155-9.

64. American College of Radiology (2013) Manual on Contrast Media. Versión 9. Disponible en: http://www.acr. org/quality-safety/resources/contrast-manual (accedido en junio de 2014).

65. Concha M, Vera C, Jara A, Atabales F, González S. Dermopatía fibrosante nefrogénica: Reporte de dos casos. Rev Med Chile 2007; 135 (5): 640-6. 